\title{
SLORT ROMUTMLGLIONS
}

\section{Accuracy of different thresholds for the anti-HIV avidity index}

\author{
Claudio Galli', Vincenzo Bossi ${ }^{2}$, Vincenza Regine ${ }^{3}$,Anna Rodella ${ }^{4}$, Nino Manca ${ }^{4}$, \\ Laura Camoni ${ }^{3}$, Barbara Suligoi ${ }^{3}$ \\ 'Medical Marketing, Abbott Diagnostici, Roma; \\ ${ }^{2}$ Laboratorio Analisi, Ospedale Amedeo di Savoia,Torino; \\ ${ }^{3}$ Dipartimento di Malattie Infettive, Parassitarie e Immunomediate, Istituto Superiore di Sanità, Roma; \\ ${ }^{4}$ Dipartimento di Microbiologia e Virologia, Università di Brescia
}

Key words: HIV, epidemiology, recent infections, antibody avidity, AxSYM

\section{Accuratezza di differenti soglie per l'indice di avidità anti-HIV}

\section{SUMMARY}

Surveillance programs for human immunodeficiency virus (HIV) infection are based on the reporting of newly diagnosed cases. In order to guarantee a more accurate estimate of the trends and behaviours of infected people, simple and reliable methods aimed at identifying recent ( $<6$ months) HIV infections are needed.

We evaluated the accuracy of the avidity index (Al) of anti-HIV antibodies on 357 serum samples obtained from 127 subjects for whom an estimated date of seroconversion was calculated on the basis of the interval between the last negative and first positive anti-HIV test result. The ROC curve analysis performed at different thresholds of the $\mathrm{Al}$ showed that a cutoff of 0.80 ( $93.0 \%$ sensitivity and $98.5 \%$ specificity) yields the best overall accuracy (95.8\%) and should be employed for surveillance purposes, whereas the application of the anti-HIV Al on individual cases is not recommended.

\section{INTRODUZIONE}

Un'analisi accurata dell'epidemia da HIV in atto nel nostro paese si dovrebbe basare sull'incidenza dell'infezione (cioè sul numero di soggetti che hanno sieroconvertito in un determinato intervallo di tempo), che indichi non soltanto la velocità di diffusione del virus ma anche le caratteristiche delle persone che si contagiano al momento attuale. Tuttavia, per conoscere l'incidenza di HIV sono necessari studi di coorte impegnativi, molto costosi e difficilmente applicabili alla popolazione generale; viceversa, i metodi di "back-calculation" a partire dai casi di AIDS, che consentivano di stimare almeno l'incidenza pregressa di HIV, non possono più essere applicati a causa dell'allungamento della sopravvivenza dei pazienti infetti (1). Si impone pertanto con crescente urgenza uno strumento che fornisca delle informazioni più attendibili sui nuovi infetti da HIV, possibilmente attraverso dei metodi semplici di diagnosi sierologica.

I sistemi di sorveglianza dell'infezione da HIV presenti in alcune nostre regioni e provincie (17) potrebbero fornire dati di incidenza, ma questi sistemi segnalano i soggetti che risultano antiHIV positivi per la prima volta e che possono quindi rappresentare vecchie infezioni non note in precedenza. Le indagini di laboratorio finora disponibili per la diagnosi e il monitoraggio dell'infezione da HIV (ricerca di anticorpi con test di "screening" e di conferma, antigene p24, test combinati per anticorpi e antigene (2), HIV-RNA, conta dei linfociti CD4) non si sono rivelate accurate nell'individuare chi, fra i nuovi infetti, fosse anche un sieroconvertito recente.

Alcuni studi internazionali hanno analizzato la possibilità di identificare le persone che hanno sieroconvertito recentemente per l'HIV attraverso un test da effettuare su un semplice campione di siero; in particolare, negli Stati Uniti è stato studiato ed applicato il "detuned test" o "STARHS" (Serologic Testing Algorithm for Recent HIV Seroconversion), che tuttavia presenta molti limiti $(7,8,11)$. Una "datazione" dell'infezione, utile sia per scopi epidemiologici (sorveglianza di infezione, incidenza) che clinici è teoricamente possibile, come per altre patologie infettive, con metodiche atte a valutare l'avidità anticorpale, in base

Corresponding author: Claudio Galli,

Medical Marketing Manager - Abbott Diagnostici - Via Mar della Cina 268 - 00144 Roma

Tel.: 06 5299|496 - Fax: 06929073019 - E-mail: claudio.galli@abbott.com 
al principio ormai assodato del progressivo incremento della medesima nei mesi successivi all'infezione primaria e della persistenza di un'avidità anticorpale elevata nelle infezioni croniche (4).

Il nostro gruppo di lavoro, con la collaborazione di esperti nel settore diagnostico e immunologico, ha messo a punto un test su siero che, valutando l'avidità degli anticorpi anti-HIV su sieri con positività anticorpale confermata, identifica le infezioni recenti ( $<6$ mesi dall'infezione) con buona accuratezza $(13,14)$. L'AI ha il vantaggio, rispetto ad altri metodi studiati per lo stesso scopo (10), di presentarsi come variabile continua consentendo così di poter scegliere diversi valori soglia a seconda dell'obiettivo prestabilito, in modo da privilegiare la sensibilità ovvero la specificità del test.

Il presente studio è stato condotto al fine di verificare quale sia la soglia ottimale dell'AI per l'identificazione delle infezioni recenti da HIV da applicare nell'ambito di un sistema di sorveglianza di tali infezioni, analizzando l'accuratezza di differenti valori soglia.

\section{MATERIALI E METODI Popolazione in studio}

Abbiamo studiato 357 campioni di siero risultati positivi agli anticorpi anti-HIV con un test EIA e confermati con Western blot. Tutti i campioni erano anonimi ed erano stati prelevati da 127 soggetti infetti; per alcuni individui erano disponibili più campioni di siero sequenziali. Abbiamo selezionato soltanto quegli individui per i quali fosse disponibile la data dell'ultimo test HIV negativo e la data del primo test positivo, necessari per stimare la data di sieroconversione. La data di sieroconversione è stata calcolata come il punto di mezzo tra le due date summenzionate.

\section{Classificazione dei campioni di siero}

Basandoci sulla data stimata di sieroconversione, abbiamo definito come 'infezioni recenti' i campioni raccolti entro 6 mesi dalla sieroconversione, mentre quelli raccolti dopo 6 mesi dalla sieroconversione sono stati considerati come 'infezioni vecchie'. Sulla base dei risultati del test di avidità abbiamo classificato i sieri con un valore di AI inferiore al cut-off presecelto come 'infezioni recenti con $\mathrm{AI}$ ', mentre quelli che avevano un valore di AI superiore al cut-off sono stati classificati come 'infezioni vecchie con AI'.

\section{Metodi di laboratorio}

I campioni di siero sono stati analizzati per l'avidità anti-HIV con il test AxSYM HIV1/2 gO (Abbott), come già descritto in precedenza (13). Brevemente, ogni campione di siero è stato suddiviso in due aliquote: una è stata pre-trattata diluendo il siero 1:10 con guanidina cloridrato
$1 \mathrm{M}$, che è un agente denaturante, mentre l'altra aliquota di siero è stata diluita 1:10 con la soluzione tampone del sistema AxSYM. Nei soggetti con infezione recente, che presentano principalmente anticorpi a bassa avidità, l'intensità del segnale emesso dall'aliquota trattata con guanidina risulta inferiore rispetto all'aliquota non trattata poiché tale agente rompe i legami deboli che si creano tra anticorpi a bassa avidità ed antigene. Il rapporto tra il segnale dell'aliquota con la guanidina e quello dell'aliquota con il tampone viene espresso come "indice di avidità" (AI), il cui valore aumenta progressivamente nel tempo con l'aumentare della maturazione degli anticorpi e raggiunge un "plateau" entro il primo anno dalla sieroconversione $(13,14)$.

\section{Analisi statistica}

La differenza tra medie è stata analizzata con il test $t$ di Student. La valutazione della sensibilità e specificità a vari livelli di AI è stata effettuata mediante l'analisi delle curve ROC (ReceiverOperating Characteristics) e confrontando le aree sottostanti le curve (6). Per gli scopi dello studio sono stati valutati quattro livelli decisionali, corrispondenti rispettivamente ai valori di AI di 0,75; 0,$80 ; 0,85$ e 0,90 .

\section{RISULTATI}

Dei 127 soggetti HIV positivi inclusi, 78 individui avevano contribuito con più di un campione di siero (due o tre sieri sequenziali). L'intervallo medio tra l'ultimo test HIV negativo ed il primo positivo era di 120.61 giorni (deviazione standard (DS) 131.68, range: 2-795 giorni) e la mediana era di 64 giorni (range interquartile: 21-187.5 giorni).

I campioni di siero sono stati prelevati tra gli anni 1985 e 2005. In base alle date stimate di sieroconversione per ciascun individuo, i campioni di siero erano stati prelevati tra 1 giorno e 10.5 anni dalla sieroconversione. In particolare, 158 $(44.3 \%)$ sieri erano stati ottenuti entro 180 giorni dalla sieroconversione e quindi definiti come infezioni recenti. Viceversa, i rimanenti 199 sieri sono stati definiti come infezioni vecchie. Più in dettaglio, 123 campioni erano stati ottenuti nei primi 3 mesi dalla sieroconversione, 35 campioni tra 4 e 6 mesi, 15 campioni tra 7 e 9 mesi, 25 tra 10 e 12 mesi, e 159 dopo un anno dalla sieroconversione. Il valore medio di AI nelle infezioni recenti era di 0,51 (DS 0,19; mediana 0,49), mentre nelle infezioni vecchie era significativamente $(\mathrm{p}<0,0001)$ più alto (media 1,00 ; DS 0,07 ; mediana 1,00$)$.

La figura I mostra la distribuzione dei valori di AI nel tempo a partire dalla data stimata di sieroconversione. La curva sovrimposta descrive con la 
massima verosimiglianza l'andamento dei valori di AI. Si osserva come l'AI aumenti rapidamente nei primi mesi dopo la sieroconversione per attestarsi ad un valore attorno all'1 dopo un anno; successivamente si mantiene costante nel tempo.

Sulla base dei risultati di AI, abbiamo analizzato la sensibilità e la specificità di AI secondo i quattro diversi cut-off considerati. I risultati ottenuti sono riportati in tabella 1 . Si osserva come, con l'innalzamento del cut-off, risultino progressivamente valori di sensibilità crescenti (da $89.2 \%$ per $\mathrm{AI}=0.75$ a $96.2 \%$ per $\mathrm{AI}=0.90)$, e di contro valori di specificità decrescenti (da $99.5 \%$ per $\mathrm{AI}=0.75 \mathrm{a}$ $92.0 \%$ per $\mathrm{AI}=0.90)$. La quota maggiore di misclassificazioni $(6.2 \%)$ viene osservata per il cut-off 0.70 e la minore $(3.9 \%)$ con il cut-off 0.80 . L'analisi delle curve ROC per i quattro valori di cut-off ha messo in evidenza come il valore di 0.80 presenti l'area maggiore sotto la curva, che peraltro è di poco superiore rispetto all'area sottostante alla curva del cut-off 0.85 . Tuttavia, il cutoff 0.80 presentava un minor numero di campioni misclassificati rispetto al cut-off 0.85 , in modo particolare tra le infezioni vecchie. Infatti, con il cut-off 0.80 l'AI ha erroneamente classificato come infezioni vecchie 11 dei 158 campioni appartenenti ad infezioni recenti; in altri termini, 11 sieri prelevati entro 180 giorni dalla data stimata di sieroconversione (infezioni recenti) mostravano un AI superiore a 0.80 . Viceversa, ha erroneamente classificato come infezioni recenti 3 dei 199 campioni appartenenti ad infezioni vecchie. Effettuando la stessa analisi con il cut-off 0.85 risulta che l'AI ha erroneamente classificato come infezioni vecchie 8 dei 158 campioni appartenenti ad infezioni recenti, mentre ha erroneamente classificato come infezioni recenti 9 dei 199 campioni appartenenti ad infezioni vecchie.

La rappresentazione grafica delle curve ROC (figura II) evidenzia chiaramente come il cut-off di 0.75 privilegi maggiormente la specificità mentre quello di 0.90 presenti alti livelli di sensibilità.

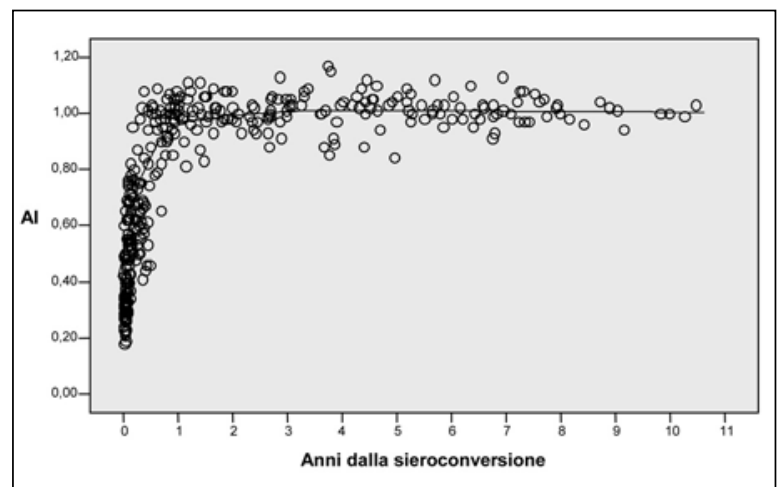

Figura I. Distribuzione temporale dei valori di Al anti-HIV dalla data stimata di sieroconversione

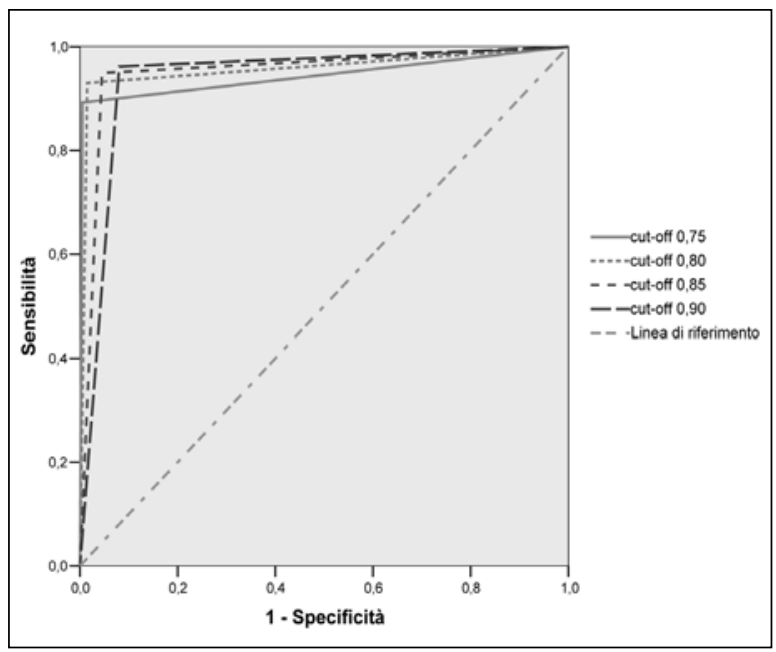

Figura II. Curve ROC per quattro diversi cut-off di Al anti-HIV

quantitativa e non qualitativa, è possibile rappresentare graficamente l'andamento di AI nel tempo: la curva disegnata in figura I riflette bene la maturazione anticorpale, presentando bassi valori di avidità degli anticorpi nei primi mesi dopo la sieroconversione, ed una stabilizzazione di tali valori nel giro di 10-12 mesi. I campioni che si discostano dalla curva sovrimposta nella figura rappresentano la variabilità biologica dei valori di AI misurati in diversi individui.

\section{DISCUSSIONE}

L'AI ha mostrato una buona capacità di distinguere le infezioni recenti da HIV da quelle vecchie, come chiaramente indicato dal valore medio di AI riscontrato nei campioni di infezioni recenti che risultava significativamente più basso di quello riscontrato nei campioni di infezioni vecchie.

Essendo l'AI una variabile
Tabella I. Sensibilità, specificità di Al e area sotto la curva ROC secondo i diversi cut-off considerati

\begin{tabular}{|c|c|c|c|c|c|c|}
\hline $\begin{array}{c}\text { Cut-off di } \\
\text { Al }\end{array}$ & $\begin{array}{c}\text { n. sieri } \\
\text { correttamente } \\
\text { identificati } \\
\text { come infezioni } \\
\text { recenti con Al }\end{array}$ & $\begin{array}{c}\text { n. sieri } \\
\text { correttamente } \\
\text { identificati } \\
\text { come infezioni } \\
\text { vecchie con Al }\end{array}$ & $\begin{array}{c}\text { n. sieri } \\
\text { misclassificati } \\
\text { con Al }\end{array}$ & Sensibilità & Specificità & $\begin{array}{c}\text { Area sotto la } \\
\text { curva ROC } \\
\text { (I.c. al 95\% } \\
\text { asintotico) }\end{array}$ \\
\hline 0.75 & 141 & 198 & 18 & $89.2 \%$ & $99.5 \%$ & $\begin{array}{c}94.4 \\
(91.5-97.3)\end{array}$ \\
\hline 0.80 & 147 & 196 & 14 & $93.0 \%$ & $98.5 \%$ & $\begin{array}{c}95.8 \\
(93.2-98.3)\end{array}$ \\
\hline 0.85 & 150 & 190 & 17 & $94.9 \%$ & $95.5 \%$ & $\begin{array}{c}95.2 \\
(92.6-97.8)\end{array}$ \\
\hline 0.90 & 152 & 183 & 22 & $96.2 \%$ & $92.0 \%$ & $(91.3-96.9)$ \\
\hline
\end{tabular}


Come precedentemente detto, essendo l'AI una variabile continua, è possibile valutarne la sensibilità e la specificità a diversi cut-off. La migliore sensibilità è stata osservata ad una soglia di 0.90 e la migliore specificità ad una soglia di 0.75 . L'equilibrio migliore tra sensibilità e specificità si rilevava per il cut-off di 0.80 , come confermato dall'analisi della curva ROC che per tale soglia presentava l'area maggiore sotto la curva. Tuttavia, la differenza rispetto ai valori rilevati per il cut-off di 0.85 era piuttosto modesta.

In definitiva, la soglia di 0.80 presentava un'accuratezza molto simile a quella di 0.85 sia in termini di sensibilità, di specificità e di area sotto la curva ROC. Tuttavia, ai fini di una sorveglianza epidemiologica appare più prudente preferire la soglia 0.80 perché presenta una specificità più alta della soglia 0.85 e quindi comporta un numero minore di falsi positivi, cioè di infezioni vecchie classificate erroneamente come recenti dal test. Come svantaggio, la minore sensibilità del cut-off 0.80 si traduce, rispetto alla soglia 0.85 , in una percentuale maggiore di falsi negativi, cioè di infezioni recenti che il test non identifica correttamente, ma che in termini numerici assoluti è piuttosto modesta poiché generalmente, in una popolazione, i soggetti HIV positivi sono di gran lunga inferiori numericamente rispetto agli HIV negativi. Queste misclassificazioni sono da attribuire ad una variabilità individuale della risposta anticorpale e della velocità di maturazione degli anticorpi specifici che non può essere prevedibile nè tantomeno artificialmente corretta in sede di analisi dei risultati. Peraltro, nell'ambito di qualsiasi analisi di variabili biologiche sono presenti dei risultati misclassificati, generalmente posti attorno ai valori del cut-off, e che determinano l'identificazione della cosiddetta "zona grigia", cioè di un range di risultati del test che non possono essere con sicurezza classificati come positivi o negativi. In definitiva, la soglia posta a 0.80 consente di ottenere una stima delle infezioni recenti più conservativa, sottostimando modicamente la percentuale vera di infezioni recenti. Per scopi epidemiologici questa soglia garantisce i risultati più accurati per l'identificazione delle infezioni recenti da HIV e per eventuali stime di incidenza dell'infezione.

Purtroppo, per nessuno dei soggetti inclusi in questo studio era stata effettuata la sottotipizzazione virale e inoltre, per garantire una maggiore protezione dei dati personali, non sono state raccolte informazioni sulla nazionalità o sui comportamenti a rischio: questo non ha consentito un'analisi dell'andamento di AI per i diversi sottotipi di HIV o in diversi sottogruppi di popolazione.

La comunicazione del risultato di questo test al singolo paziente ci sembra sconsigliabile in quanto la pur limitata probabilità di fornire una risposta non corretta potrebbe avere serie conseguenze dal punto di vista psicologico o medico-legale.

Per quanto riguarda gli aspetti operativi, l'AI è un test poco costoso (include il costo di due test di screening e della guanidina), di semplice esecuzione, non richiede né un particolare addestramento del personale di laboratorio, né apparecchiature sofisticate; necessita soltanto di una prediluizione manuale del siero mentre il resto della procedura è automatizzata, garantendone la riproducibilità e non necessita di calibratori. Al momento attuale i test AxSYM HIV sono utilizzati in 88 nazioni in tutto il mondo nei cinque continenti (C. Galli, comunicazione personale, 2006), il che rende questo test facilmente esportabile in altre nazioni consentendo così di poter confrontare i risultati ottenuti in diverse aree geografiche e in diversi laboratori. Rispetto al test STARHS, largamente utilizzato solo negli Stati Uniti, l'AI ha mostrato una maggiore specificità, riproducibilità e semplicità di esecuzione (9).

Il razionale su cui si basa l'AI, cioè la maturazione dell'avidità anticorpale dopo l'infezione, non è strettamente vincolato al tipo di test usato in questo studio e può essere trasferito ad altri formati di test HIV mantenendo la medesima accuratezza. Questo è particolarmente importante in vista dell'adozione sempre più diffusa dei test cosiddetti di "quarta generazione" che consentono la determinazione simultanea di anticorpi anti-HIV e dell'antigene p24 (5).

In base ai nostri dati, e in ragione della buona precisione della procedura (13), si può ritenere che l'applicazione di un cut-off di 0.80 sia appropriato per studi epidemiologici e per l'applicazione in sistemi di sorveglianza delle infezioni da HIV allo scopo di stimare e monitorare l'incidenza dell'infezione da HIV (3). Il dato di incidenza, infatti, fornisce le informazioni più accurate sulla diffusione attuale del virus, sulle popolazioni ed aree a maggior rischio di infezione, e di conseguenza sulle necessità più urgenti in termini di prevenzione ed assistenza medica. Infine, questo test può essere utilizzato per valutare l'efficacia di programmi di prevenzione primaria (vaccinazione) e secondaria che mirano ad una diminuzione dell'incidenza dell'infezione. 


\section{BIBLIOGRAFIA}

1. (no authors listed) Time from HIV-1 seroconversion to AIDS and death before widespread use of highlyactive antiretroviral therapy: a collaborative re-analysis. Collaborative Group on AIDS Incubation and HIV Survival including the CASCADE EU Concerted Action on SeroConversion to AIDS and Death in Europe. Lancet 2000, 355: 1131-7.

2. Andersson S, Asjo B, Jenum PA, Manner I, et al. Relevance of a combined HIV antigen/ antibody assay to detect early HIV infections in a low prevalence population: case reports. Clin Lab 2004; 50: 109-13.

3. Centers for Disease Control and Prevention. Twentyfive years of HIV/AIDS - United States, 1981-2006. MMWR 2006; 55: 585-603.

4. Eisen HN, Siskind GW. Variations in affinities of antibodies during the immune response. Biochemistry 1964; 7: 996-1008.

5. Galli C, Bossi V. Valutazione dell'indice di avidità anti-HIV con due dosaggi automatizzati. $2^{\circ}$ Congresso Nazionale SIVIM; Roma, 16-18 maggio 2005, poster n. 54 .

6. Hanley J, McNeil BJ. The meaning and use of the area under a receiver operating characteristic (ROC) curve. Radiology 1982; 143: 29-36.

7. Janssen RS, Satten GA, Stramer SL, et al. New testing strategy to detect early HIV-1 infection for use in incidence estimates and for clinical and prevention purposes. JAMA 1998; 280: 42-8.

8. Kothe D, Byers RH, Caudill SP, et al. Performance characteristics of a new less sensitive HIV-1 enzyme immunoassay for use in estimating HIV seroincidence. J Acquir Immune Defic Syndr 2003 Aug 15; 33(5): 625-34.

9. Martrò E, Suligoi B, Gonzalez V, et al. Comparison of the avidity index method and the serologic testing algorithm for recent human immunodeficiency virus (HIV) seroconversion, two methods using a single serum sample for identification of recent HIV infections. J Clin Microbiol 2005; 43: 6197-99.

10. Parekh BS, McDougal JS. Application of laboratory methods for estimation of HIV-1 incidence. Indian J Med Res 2005; 121: 510-8.

11. Schwarcz S, Kellogg T, McFarland W, et al. Differences in the temporal trends of HIV seroincidence and seroprevalence among sexually transmitted disease clinic patients, 1989-1998: application of the serologic testing algorithm for recent HIV seroconversion. Am J Epidemiol 2001; 153: 925-34.

12. Suligoi B, Boros S, Camoni L, Lepore D, Ferri M, Roazzi P. Aggiornamento dei casi di AIDS e delle nuove diagnosi di HIV in Italia. Notiziario dell'Istituto Superiore di Sanità 2006, 19 (6), suppl 1: $3-23$.

13. Suligoi B, Galli C, Massi M, et al. The precision and accuracy of a procedure for detecting recent HIV infections, calculating the antibody avidity index using an automated immunoassay. J Clin Microbiol 2002; 40: 4015-20.

14. Suligoi B, Massi M, Galli C, et al. Identifying recent HIV infections using the avidity index and an automated enzyme immunoassay. J Acq Immun Def Synd 2003; 32: 424-8. 